\title{
P I I-05. Induction of CD8+ T cell mediated immune responses through skin and mucosa: identification of immunostimulatory versus tolerogenic dendritic cells
}

\author{
B Dubois, M Gomez de Agüero, M Le Borgne, M Gouanvic, M Vocanson and \\ D Kaiserlian*
}

Address: INSERM U851, Lyon, France

* Corresponding author

from AIDS Vaccine 2009

Paris, France. 19-22 October 2009

Published: 22 October 2009

Retrovirology 2009, 6(Suppl 3):PI50 doi:10.I I86/I742-4690-6-S3-PI50

This abstract is available from: http://www.retrovirology.com/content/6/S3/PI50

(C) 2009 Dubois et al; licensee BioMed Central Ltd.

\section{Background}

Mucosae and skin are populated with dendritic cells (DC) (epithelial Langerhans cells [LC] and conventional DC in lamina propria/dermis) and represent privileged sites for anti-infectious vaccination.

\section{Methods}

To determine which immunostimulatory DC subset needs be targeted by vaccines, we investigated the nature of DC involved in the induction or regulation of cytotoxic CD8+ T cells (CTL) in mice.

\section{Results}

We first documented (Leborgne et al. Immunity, 2006) that CD8+ CTL can be primed by intradermal immunization (in buccal mucosa or skin) with a protein Ag combined with adjuvants inducing local secretion of the chemokine CCL20. We found that in vivo priming of CTL responses was induced by newly recruited DC rather than skin/mucosal resident DC. These inflammatory-type DC derive from circulating Gr1+ monocytes, are recruited by a process involving CCL20 and CCR6, and directly crosspresent the Ag to CD8+ T cells. Alternatively, using conditional ablation of LC in Langerin-DTR mice and the contact sensitizer DNFB (which modifies self proteins), we showed that epithelial LC are dispensable for priming CD8+ CTL mediating delayed-type hypersensitivity responses. Moreover, using a structurally related but tolerogenic hapten, DNTB, we demonstrated that LC migrate to draining lymph nodes for Ag presentation to CD8+ T cells, but were unable to prime CTL. Depletion of LC prior to hapten immunization allowed for priming of cytotoxic $\mathrm{T}$ cells, indicating a tolerogenic role for LC. In addition, induction of potent CTL responses is accompanied by the recruitment of inflammatory monocytes at the site of immunization and presentation of the $\mathrm{Ag}$ in draining lymph nodes by both LC and Langerin-DC.

\section{Conclusion}

Our data demonstrate that, while resident LC of pluristratified mucosae and skin display tolerogenic functions, adjuvants able to recruit monocyte-derived inflammatory DC into mucosae can break tolerance and allow for priming of protective cytotoxic CD8+ T cells. 